\title{
Neurological Manifestations of Patients Infected with COVID-19: A Systematic Review
}

\author{
Jafari $\mathbf{N}^{1 *}$, Khoshghamat N ${ }^{1 *}$, Ghaderi MS ${ }^{2 *}$, \\ Yazdani S', Sepehrinezhad $A^{3,4}$, Saburi $E^{5}$, Hajali \\ $V^{4}$ and Sahab Negah $S^{4,6,7,8 *}$ \\ ${ }^{1}$ Student Research Committee, Faculty of Medicine, \\ Mashhad University of Medical Sciences, Mashhad, Iran \\ ${ }^{2}$ Student Research Committee, School of Nursing and \\ Midwifery, Mashhad University of Medical Sciences, \\ Mashhad, Iran \\ ${ }^{3}$ Department of Neuroscience, Faculty of Advanced \\ Technologies in Medicine, Iran University of Medical \\ Sciences, Tehran, Iran \\ ${ }^{4}$ Neuroscience Research Center, Mashhad University of \\ Medical Sciences, Mashhad, Iran \\ ${ }^{5}$ Medical Genetics and Molecular Medicine Department, \\ School of Medicine, Mashhad University of Medical \\ Sciences, Mashhad, Iran \\ ${ }^{6}$ Shefa Neuroscience Research Center, Khatam Alanbia \\ Hospital, Tehran, Iran \\ ${ }^{7}$ Department of Neuroscience, Faculty of Medicine, \\ Mashhad University of Medical Sciences, Mashhad, Iran \\ ${ }^{8}$ Society for Brain Mapping and Therapeutics, Iranian \\ Chapter, SBMT, Los Angeles, USA \\ "Contributed equally to this work
}

*Corresponding author: Sajad Sahab Negah, Neuroscience Research Center, Mashhad University of Medical Sciences, Pardis Campus, Azadi Square, Kalantari Blvd., Mashhad, Iran

Received: June 15, 2021; Accepted: July 09, 2021;

Published: July 16, 2021

\begin{abstract}
In the light of the recent evidence, neurological manifestations have been reported in patients infected with Severe Acute Respiratory Syndrome Coronavirus 2 (SARS-CoV-2). To shed light on a clear-cut explanation of neurological complications followed by SARS-CoV-2, a systematic analysis was reviewed. To this point, general medical databases, such as PubMed, Scopus, and Google Scholar from January 1, 2020, to September 20, 2020, were systematically searched to find related published articles. Through the search strategy, 93 studies for qualitative synthesis were included. Finally, the available data of 8,753 hospitalized patients with coronavirus disease 2019 (COVID-19) showed that the most prevalent Central Nervous System (CNS) manifestations of COVID-19 were headache (1137 cases, 18\%) and dizziness (142 cases, 2\%). On the other hand, some peripheral nervous system (PNS) symptoms were muscle pain (3300 cases, 53\%), hypogeusia (646 cases, 11\%), anosmia (545 cases, $9 \%$ ), and hyposmia (379 cases, 6\%). Furthermore, a small proportion of patients had ageusia (74 cases, 1\%), cerebrovascular disorders (66 cases, 0.75\%), and impaired consciousness (21 cases, 0.2\%). Besides, some neurological features, such as encephalitis, neuralgia, ataxia, GuillainBarre syndrome, miller fisher syndrome, intracerebral hemorrhage, polyneuritis cranialis, and dystonic posture were reported in the literature as case reports and case series.
\end{abstract}

Conclusion: Taken together, data from our systematic analysis indicated that neurological manifestations in both CNS and PNS were reported in COVID-19 patients. Further observational research in this field with more participants are needed to detect the specific risk concerning neurological complications in COVID-19 infection.

Keywords: COVID-19; Neurological manifestation; Systematic review

\section{Abbreviations}

ACE2: Angiotensin-Converting Enzyme-2; CNS: Central Nervous System; COVID-19: Coronavirus Disease 2019; PCR: Polymerase Chain Reaction; PNS: Peripheral Nervous System; PRISMA: Preferred Reporting Items for Systematic Reviews and Meta-Analyses; SARS-CoV-2: Severe Acute Respiratory Syndrome Coronavirus 2; TNF: Tumor Necrosis Factor; WHO: World Health Organization.

\section{Introduction}

In December 2019, Coronavirus Disease 2019 (COVID-19) caused by Severe Acute Respiratory Syndrome Coronavirus 2 (SARS-CoV-2) was reported for the first time in Wuhan, China, and then spread rapidly out to the rest of the world [1]. The COVID-19 outbreak has been announced a pandemic by the World Health Organization. The major clinical symptoms of COVID-19 are associated with respiratory and cardiovascular systems. The most accepted hypothesis for the pathogenesis of SARS-CoV-2 is the interaction between Angiotensin-Converting Enzyme-2 (ACE2) receptor and SARS-CoV-2 [2,3]. SARS-CoV-2 can infect and destroy host target cells through the ACE2 receptor. ACE2 receptor expresses on the surface of many types of human cells, such as the lungs, heart, gut, and brain [4,5]. Regard to this pathogenesis, it has been suggested that ACE2 acts as the main host receptor for the SARS-CoV-2 virus in the CNS and facilitates its neuroinvasion [6]. On the other hand, clinical observational studies have been reported that about $36 \%$ of COVID-19 patients have neurological symptoms [7,8]. Determination of neurological complications followed by SARS-CoV-2 infection is necessary because CNS viral infections may cause long-lasting brain injury [3]. Therefore, conducting systematic reviews on neurological manifestations infected with SARS-CoV-2 can guide neuroscientists to find risk factors and help physicians to manage the treatment of COVID-19 patients. In this systematic review, we comprehensively reported various neurological manifestations in COVID-19 patients.

\section{Methods}

\section{Search strategy}

This systematic review was administered on papers published from January 1, 2020, to September 20, 2020, in regular databases including PubMed, Scopus, and Google Scholar. We searched the combination of keywords related to neurological diseases, neurological manifestations, CNS and PNS symptoms, and COVID-19 that were used with Boolean operators for more efficient literature retrieval
Austin J Neurol Disord Epilepsy - Volume 7 Issue 1 - 2021 ISSN: 2472-3711 | www.austinpublishinggroup.com Negah et al. (C) All rights are reserved
Citation: Jafari N, Khoshghamat N, Ghaderi MS, Yazdani S, Sepehrinezhad A, Saburi E, et al. Neurological Manifestations of Patients Infected with COVID-19: A Systematic Review. Austin J Neurol Disord Epilepsy. 2021; 7(1): 1046. 


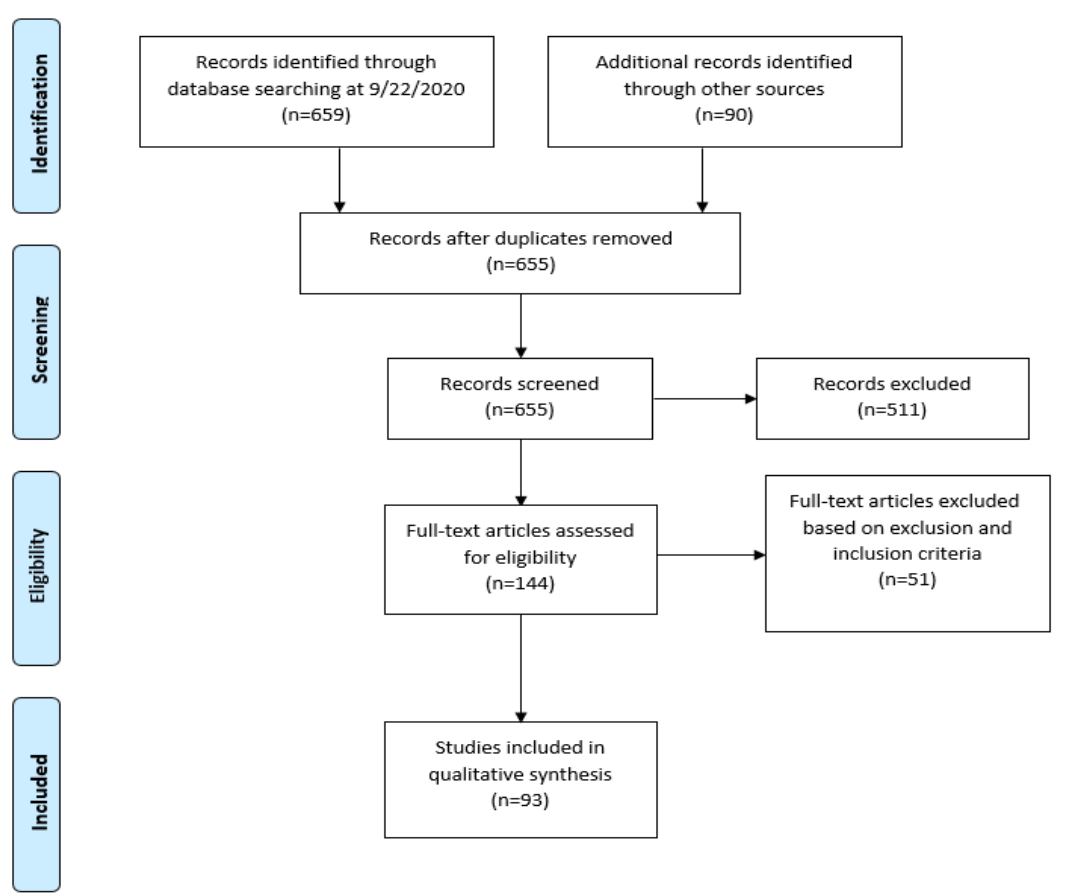

Figure 1: The PRISMA flow diagram of database search.

(Supplementary 1).

To assure the validity and accuracy of our study, we investigated the reference list for each selected article to recognize possible missing articles. We expanded our search manually to recognize additional related articles. No language limitations were imposed. The search terms used in this study were Nervous System, Central Nervous System Diseases, Neurologic Signs and Symptoms, Brain Disorders, Headache, Anosmia, Neurologic Manifestations, Cerebrovascular Disease, Coronavirus, COVID-19, and SARS-CoV-2.

\section{Study selection}

The searching was performed by three authors (M.S.G, N.KH, and N.J). Duplication results were removed by the EndNote (X8 software). Additional investigators independently screened studies for the inclusion criteria. Our systematic review was assessed according to the Preferred Reporting Items for Systematic Reviews and Meta-Analyses (PRISMA) checklist.

\section{Inclusion and exclusion criteria and study selection}

We included original studies (e.g. Case-Control, Cross-Sectional, Cohort, Case-Series, and Case Report) that reported neurological manifestations in COVID-19 patients, which confirmed with World Health Organization (WHO) interim guidance [9] and polymerase chain reaction (PCR). We excluded all letters, comments, review articles, and communications. We also excluded studies that did not report a reliable test for confirmation of SARS-CoV-2.

\section{Data extraction}

The extraction table contains information as follows: databases, title, journal, hospital, first author, publication date, location, patient data, patient source, sample size, study type, age, gender, CNS symptoms (headache, dizziness, and impaired consciousness),
PNS symptoms (hypogeusia, anosmia, hyposmia, and myalgia), neurodegenerative diseases, and laboratory brain test. All investigators extracted the data from selected articles. Any possible disagreement was resolved by consensus.

\section{Results}

\section{Literature search}

The PRISMA flow diagram of database search was summarized in Figure 1. In the initial search, 749 reports were screened for the analysis of patients with COVID-19. Based on the PRISMA checklist, 94 articles were excluded because of duplication, 511 were excluded after the title and abstract screening, and the full text of 144 reports was reviewed, after that 51 full-text articles didn't meet inclusion criteria and were removed. Finally, 93 studies met the inclusion criteria (Figure 1).

\section{Characteristics of included studies}

The characteristics of the included studies are shown in Table 1. Studies extracted were case report $(41,44 \%)$, cross-sectional retrospective $(28,30 \%)$, case series $(12,13 \%)$, cohort $(11,12 \%)$, and case-control $(1,1.1 \%)$ (Table 1$)$. All studies were published in 2020 and the majority of reports $(41,44 \%$ of studies) were from China. Finally, the available data of 8,753 hospitalized patients with COVID-19 infection were used for this systematic review. Three reports have not mentioned gender in the analysis of patients with COVID-19.

\section{Neurological manifestations of COVID-19 patients}

Overall, 8,753 confirmed patients with COVID-19 infection were included in the review, which showed that 4,329 patients (53\%) were male. The most prevalent CNS symptoms were headache (1137 cases, 18\%) and dizziness (142 cases, 2\%) and the most prevalent PNS 
Table 1: Characteristics of the studies and nervous symptoms included in the systematic review.

\begin{tabular}{|c|c|c|c|c|c|c|c|c|c|c|c|c|c|c|c|}
\hline \multirow[b]{2}{*}{ First Author } & \multirow[b]{2}{*}{ Study Type } & \multirow[b]{2}{*}{\begin{tabular}{|} 
Publication \\
Date (MMI \\
DD)
\end{tabular}} & \multirow[b]{2}{*}{ Location } & \multirow[b]{2}{*}{$\begin{array}{c}\text { Sample } \\
\text { Size }\end{array}$} & \multirow[b]{2}{*}{$\begin{array}{l}\text { Age: Mean } \pm \text { SDI } \\
\text { Median (IQR) } \\
\text { (year) }\end{array}$} & \multirow[b]{2}{*}{$\begin{array}{l}\text { Gender } \\
\text { (Malel } \\
\text { Female) }\end{array}$} & \multicolumn{4}{|c|}{ Central Nervous System (CNS) Symptoms } & \multicolumn{5}{|c|}{ Peripheral Nervous System (PNS) Symptoms } \\
\hline & & & & & & & Dizziness & Headache & $\begin{array}{c}\text { Impaired } \\
\text { consciousness }\end{array}$ & $\begin{array}{c}\text { Cerebrovascular } \\
\text { diseases }\end{array}$ & $\begin{array}{c}\text { Muscle } \\
\text { ache or } \\
\text { fatigue or } \\
\text { myalgia }\end{array}$ & \begin{tabular}{|c|} 
Smell \\
disorders \\
(including \\
hyposmia)
\end{tabular} & Anosmia & \begin{tabular}{|c|} 
Taste \\
disorders \\
(including \\
hypogeusia)
\end{tabular} & Ageusia \\
\hline Gupta [22] & $\begin{array}{c}\text { Retrospective } \\
\text { observational case } \\
\text { series }\end{array}$ & $4 / 6$ & New dehli, India & 21 & 40.3 (16-73 IQR) & $14 / 7$ & & 3 & & & & & & & \\
\hline $\mathrm{He}[23]$ & Retrospective study & $4 / 5$ & Wuhan, China & 204 & 49 (34-62IQR) & $79 / 125$ & & 14 & & & 66 & & & & \\
\hline Huang [24] & Retrospective study & $4 / 15$ & Wuhan, China & 41 & $49(41-58)$ & $30 / 11$ & & 3 & & & 18 & & & & \\
\hline $\operatorname{Jin}[25]$ & Retrospective study & $3 / 17$ & Zhejiang, China & 651 & 45.2 & $331 / 320$ & & 67 & & & 190 & & & & \\
\hline Kong [26] & Retrospective study & $2 / 21$ & South Korea & 28 & $42.6(20-73)$ & $15 / 13$ & & 3 & & & 7 & & & & \\
\hline Liu [27] & Retrospective study & $2 / 7$ & $\begin{array}{l}\text { Hubei Province, } \\
\text { China }\end{array}$ & 137 & $55 \pm 16$ & $61 / 76$ & & 13 & & & 44 & & & & \\
\hline Liu [28] & $\begin{array}{l}\text { Retrospective cohort } \\
\text { study }\end{array}$ & $4 / 3$ & Wuhan, China & 245 & $53.95 \pm 16.90$ & $114 / 131$ & 10 & 12 & & & 192 & & & & \\
\hline Lu [29] & Retrospective study & $4 / 10$ & Wuhan, China & 141 & $49(9-87)$ & $77 / 64$ & & 11 & & & & & & & \\
\hline Qin [30] & Cohort study & $3 / 12$ & Wuhan, China & 452 & $58(47-67)$ & $235 / 217$ & 37 & 52 & & 11 & 310 & & & & \\
\hline Tian [31] & Retrospective study & $2 / 21$ & $\begin{array}{l}\text { Beijing/Wuhan, } \\
\text { China }\end{array}$ & 262 & $47.5(1-94)$ & $127 / 135$ & & 17 & & & 69 & & & & \\
\hline Yan [32] & Cross sectional & $4 / 12$ & California, USA & 59 & $48.5(18-79)$ & $\begin{array}{c}\text { 29/29 } \\
\text { Gender } \\
\text { Diverse:1 }\end{array}$ & & 39 & & & 85 & & 40 & & 42 \\
\hline Zhang [33] & $\begin{array}{l}\text { Single center, } \\
\text { retrospective, } \\
\text { Observational study }\end{array}$ & $4 / 1$ & Wuhan, China & 120 & $45.4 \pm 15.6$ & $43 / 77$ & & 28 & & & 57 & & & & \\
\hline $\begin{array}{c}\text { Beltrán } \\
\text { Corbellini [34] }\end{array}$ & Cross sectional & $4 / 22$ & Madrid, Spain & 79 & $61.6 \pm 17.4$ & $48 / 31$ & & & & & & 25 & 14 & 28 & 14 \\
\hline Bénézit [35] & Cross sectional & $4 / 15$ & western France & 259 & Not mentioned & $\begin{array}{c}\text { Not } \\
\text { mentioned }\end{array}$ & & & & & & 51 & & 63 & \\
\hline Chen [36] & $\begin{array}{l}\text { Case series (single } \\
\text { center retrospective } \\
\text { observational study) }\end{array}$ & $4 / 28$ & Taizhou, China & 145 & $47.5 \pm 14.6$ & $79 / 66$ & 29 & 24 & & & 79 & & & & \\
\hline Chen [37] & Case series & $3 / 26$ & Wuhan, China & 274 & $62.0(44.0-70.0)$ & $171 / 103$ & 21 & 31 & & 4 & 60 & & & & \\
\hline Klopfenstein [38] & Cross sectional & $4 / 17$ & Trévenans, France & 114 & Not mentioned & $\begin{array}{c}\text { Not } \\
\text { mentioned }\end{array}$ & & 44 & & & 133 & & 54 & & \\
\hline Lechien [39] & Cross sectional & $4 / 18$ & France & 78 & $40.6 \pm 11.2$ & $32 / 46$ & & & & & & 11 & 24 & 53 & \\
\hline Lechien [40] & Cross sectional & $4 / 6$ & $\begin{array}{c}\text { Belgium } \\
\text { Brussels } \\
\text { Mons } \\
\text { Baudour Ath } \\
\text { France }\end{array}$ & 417 & $36.9 \pm 11.4$ & $154 / 263$ & & 188 & & & 242 & 73 & 284 & 342 & \\
\hline Wang [41] & Cohort & $3 / 16$ & Wuhan, China & 69 & $42.0(35.062 .0)$ & $32 / 37$ & 5 & 10 & & & 50 & & & & \\
\hline Vaira [42] & Cross sectional & $4 / 27$ & Sassari, Italy & 72 & $49.2 \pm 13.7$ & $27 / 45$ & & 30 & & & & 44 & & 39 & \\
\hline Lu [43] & $\begin{array}{c}\text { Retrospective } \\
\text { multicenter study cohort }\end{array}$ & $4 / 18$ & $\begin{array}{c}\text { Sichuan province, } \\
\text { China }\end{array}$ & 304 & $44(33 \pm 59.25)$ & $182 / 122$ & & & & 3 & & & & & \\
\hline Mao [1] & $\begin{array}{c}\text { Retrospective, } \\
\text { observational case } \\
\text { series }\end{array}$ & $4 / 10$ & Wuhan, China & 214 & $52.7 \pm 15.5$ & $87 / 127$ & 36 & 28 & 16 & 15 & 23 & 11 & & 12 & \\
\hline Mi [44] & $\begin{array}{l}\text { Retrospective case } \\
\text { series }\end{array}$ & $4 / 1$ & Wuhan, China & 6 & 72.16 & $2 / 4$ & 2 & & & & 5 & & & & \\
\hline Moein [45] & Case control & $4 / 17$ & Tehran, Iran & 60 & $46.55 \pm 12.17$ & $40 / 20$ & & 22 & & & 5 & 44 & 15 & & 14 \\
\hline Tostmann [46] & Cohort & $4 / 23$ & Netherlands & 90 & 39.5 & $19 / 71$ & & 64 & & & 57 & 37 & & & \\
\hline Li [47] & Cross sectional & $2 / 23$ & Wuhan, China & 31 & $54 \pm 13$ & $15 / 16$ & & 8 & & & 32 & & & & \\
\hline Liu [48] & Cross sectional & $2 / 17$ & Jianghan, China & 30 & $35 \pm 8$ & $10 / 20$ & & 16 & & & 21 & & & & \\
\hline Sun [49] & Cross sectional & $4 / 15$ & Nanyang City & 150 & $45 \pm 16$ & $67 / 83$ & & & & 2 & 23 & & & & \\
\hline Chen [50] & Case Series & $1 / 29$ & Wuhan, China & 99 & $55 \cdot 5 \pm 13.1(21-82)$ & $67 / 32$ & & 8 & & & 11 & & & & \\
\hline Ding [51] & Case series & $3 / 20$ & Wuhan, China & 5 & $\begin{array}{l}50.20 \pm 9.83 \\
(3966)\end{array}$ & $2 / 3$ & & 2 & & & 4 & & & & \\
\hline Du [52] & Prospective Cohort & $4 / 8$ & Wuhan, China & 179 & $57.6 \pm 13.7(18-87)$ & $97 / 82$ & & 17 & & & 105 & & & & \\
\hline $\begin{array}{c}\text { Escalera- } \\
\text { Antezana [53] }\end{array}$ & $\begin{array}{c}\text { Observational, } \\
\text { retrospective and Cross } \\
\text { sectional }\end{array}$ & $3 / 27$ & $\begin{array}{l}\text { Bolivia, South } \\
\text { American }\end{array}$ & 12 & $39(25-43)$ & $6 / 6$ & & & & & 5 & & & & \\
\hline Gilani [54] & Case series & $4 / 23$ & Tehran, Iran & 5 & $39(35-44)$ & 3-Feb & & & & & 1 & & 5 & & \\
\hline Wang [55] & Retrospective & $3 / 2$ & China & 31 & $7(0.5-17)$ & $15 / 16$ & & 3 & & & 3 & & & & \\
\hline Wang [56] & Retrospective & $4 / 9$ & Wuhan, China & 57 & $40(33-49)$ & $25 / 32$ & & 7 & & 1 & 40 & & & & \\
\hline $\mathrm{Xu}[57]$ & $\begin{array}{l}\text { Retrospective case } \\
\text { series }\end{array}$ & $4 / 14$ & $\begin{array}{c}\text { Zhejiang province, } \\
\text { China }\end{array}$ & 62 & $41(32-52)$ & $36 / 27$ & & 21 & & & 32 & & & & \\
\hline
\end{tabular}




\begin{tabular}{|c|c|c|c|c|c|c|c|c|c|c|c|c|c|c|}
\hline Yan [32] & $\begin{array}{l}\text { Retrospective cohort } \\
\text { study }\end{array}$ & $4 / 24$ & San Diego, USA & 128 & \begin{tabular}{|c|} 
admitted 53.5 \\
(40-65) outpatient \\
43.0 (34-54) \\
\end{tabular} & $61 / 67$ & & 62 & & & 90 & & 75 & 70 \\
\hline Yan [58] & $\begin{array}{l}\text { Single center, } \\
\text { retrospective, } \\
\text { observational }\end{array}$ & $4 / 27$ & Wuhan, China & 193 & $64(49-73)$ & $114 / 79$ & & 21 & & 8 & 101 & & & \\
\hline Zhang [59] & Case series & $4 / 2$ & Wuhan, China & 5 & $45 \pm 11$ & $4 / 1$ & & & & & 3 & & & \\
\hline Zhang [60] & Retrospective & $3 / 20$ & Zhejiang, China & 645 & \begin{tabular}{|c|} 
Normal imaging \\
$34.90 \pm 14.20$ \\
Abnormal imaging \\
$46.65 \pm 13.82$ \\
\end{tabular} & $328 / 317$ & & 67 & & & 189 & & & \\
\hline Zheng [61] & $\begin{array}{l}\text { Retrospective, single } \\
\text { center case series }\end{array}$ & $4 / 10$ & Chengdu, China & 99 & \begin{tabular}{|c|}
$49.4 \pm 18.45(0.25-$ \\
$87.00)$
\end{tabular} & $51 / 48$ & & 12 & & & 72 & & & \\
\hline Zhang [62] & $\begin{array}{l}\text { Retrospective cohort } \\
\text { study }\end{array}$ & $3 / 26$ & Wuhan, China & 28 & $65.0(56.0-70.0)$ & $17 / 11$ & & & & 15 & 22 & & & \\
\hline Monti [63] & Case series & $4 / 2$ & Pavia, Italy & 4 & $58 \pm 5$ & 4 Female & & 2 & & & 4 & & 3 & 3 \\
\hline Guan [64] & Cohort & 28-Feb & $\begin{array}{c}\text { Guangzhou, Wuhan, } \\
\text { China }\end{array}$ & 1099 & $47.0(35.0-58.0)$ & $637 / 459$ & & 150 & & 7 & 583 & & & \\
\hline Zhou [65] & Cohort & $4 / 3$ & Wuhan, China & 191 & $56 \cdot 0(46 \cdot 0-67 \cdot 0)$ & $119 / 72$ & & & & & 73 & & & \\
\hline Wu [66] & Cohort & $3 / 13$ & Wuhan, China & 201 & $51(43-60)$ & $128 / 73$ & & & & & 65 & & & \\
\hline Mo [67] & Case series & $3 / 17$ & Wuhan, China & 155 & $54(42-66)$ & $86 / 69$ & 2 & 8 & & & 110 & & & \\
\hline Hintschich [68] & Prospective & $7 / 1$ & $\begin{array}{l}\text { Regensburg, } \\
\text { Germany }\end{array}$ & 41 & 37 & $12 / 29$ & & & & & & 25 & 18 & \\
\hline Coppola [69] & $\begin{array}{c}\text { Observational } \\
\text { Prospectical Study }\end{array}$ & $9 / 11$ & Milanese, Italy" & 73 & 69.75 & $52 / 21$ & & & & & & & & \\
\hline Klok [70] & $\begin{array}{l}\text { Retrospective Cohort } \\
\text { Study }\end{array}$ & $4 / 10$ & $\begin{array}{l}\text { Leiden, the } \\
\text { Netherlands }\end{array}$ & 184 & 64 (12) & $139 / 45$ & & & & & & & & \\
\hline Oxley [71] & Case report & $4 / 28$ & $\begin{array}{l}\text { New York, united } \\
\text { states }\end{array}$ & 5 & 40.4 & $4 / 1$ & & & 4 & & 5 & 22 & & 8 \\
\hline Helms [72] & $\begin{array}{l}\text { Observational, } \\
\text { Retrospective }\end{array}$ & $6 / 4$ & Strasbourg, France & 58 & 63 & $\begin{array}{c}\text { Not } \\
\text { mentioned }\end{array}$ & & & & & & & & \\
\hline Garg [73] & Observational Study & $8 / 14$ & $\begin{array}{l}\text { Madhya Pradesh, } \\
\text { India }\end{array}$ & 391 & $49.07 \pm 15.74$ & $241 / 150$ & & 24 & & & & 35 & 8 & 26 \\
\hline $\begin{array}{c}\text { Camdessanche } \\
{[21]}\end{array}$ & Case Report & $7 / 12$ & $\begin{array}{l}\text { Saint Etienne, } \\
\text { France }\end{array}$ & 1 & 64 & Male & & & & & & & & \\
\hline Jang [74] & Case Report & $7 / 12$ & Seoul, Korea & 1 & 42 & Male & & & & & 1 & & 1 & 1 \\
\hline $\begin{array}{c}\text { Ollarves Carrero } \\
\text { [75] }\end{array}$ & Case Report & $4 / 7$ & Madrid, Spain & 1 & 40 & Female & & 1 & & & 1 & & 1 & \\
\hline Zhao [76] & Case Report & $4 / 1$ & Shanghai, China & 1 & 61 & Male & & & & & & & & \\
\hline Moriguchi [11] & Case Report & $3 / 25$ & Yamanashi, Japan & 1 & 24 & Male & & 1 & & & 1 & & & \\
\hline Sedaghat [77] & Case Report & $4 / 11$ & Sari, Iran & 1 & 65 & Male & & & & & & & & \\
\hline ElOtmani [78] & Case Report & $4 / 20$ & $\begin{array}{l}\text { Casablanca, } \\
\text { Morocco }\end{array}$ & 1 & 70 & Female & & & & & & & & \\
\hline Huang [79] & Case Report & $5 / 6$ & $\begin{array}{l}\text { Los Angeles, United } \\
\text { States }\end{array}$ & 1 & 40 & Female & & & & & & & & \\
\hline Duong [80] & Case Report & $4 / 17$ & $\begin{array}{c}\text { Los Angeles, United } \\
\text { States }\end{array}$ & 1 & 41 & Female & & & & & & & & \\
\hline Ye [81] & Case Report & $4 / 10$ & Wuhan, China & 1 & & Male & & & & & & & & \\
\hline Yin [82] & Case Report & $4 / 15$ & $\begin{array}{l}\text { Wuhan, Hubei, } \\
\text { China }\end{array}$ & 1 & 64 & Male & & & & & & & & \\
\hline Filatov [83] & Case Report & $5 / 21$ & $\begin{array}{c}\text { Boca Raton, United } \\
\text { states }\end{array}$ & 1 & 74 & Male & & & & & 1 & & 1 & 1 \\
\hline Wong [84] & Case Report & $4 / 7$ & Telford, UK & 1 & 40 & Male & & 1 & & & 1 & & 1 & \\
\hline Virani [85] & Case Report & $4 / 12$ & $\begin{array}{l}\text { Pittsburgh, United } \\
\text { states }\end{array}$ & 1 & 54 & Male & & & & & & & & \\
\hline Alberti [86] & Case Report & $3 / 25$ & Milan, Italy & 1 & 71 & Male & & 1 & & & 1 & & & \\
\hline Scheidl [87] & Case Report & $4 / 11$ & Selters, Germany & 1 & 54 & Female & & & & & & & & \\
\hline Ottaviani [88] & Case Report & $5 / 12$ & Trento, Italy & 1 & 66 & Female & & & & & & & & \\
\hline Abdelnour [89] & Case Report & $4 / 27$ & $\begin{array}{l}\text { Northern Ireland, } \\
\text { United Kingdom }\end{array}$ & 1 & 69 & Male & & & & & & & & \\
\hline $\begin{array}{c}\text { Caamaño DS } \\
{[90]}\end{array}$ & Case Report & $5 / 14$ & Madrid, Spain & 1 & 61 & Male & & & & & & & & \\
\hline Wei [91] & Case Report & $2 / 26$ & Wuhan, China & 1 & 62 & Male & & & & & & & & \\
\hline Jin [92] & Case Report & $6 / 21$ & Wuhan, China & 1 & 61 & Female & & & & & & & & \\
\hline $\begin{array}{c}\text { Suwanwongse } \\
{[93]}\end{array}$ & Case Report & $4 / 6$ & New York City, USA & 1 & 88 & Male & & & & & & & & \\
\hline Vollono [94] & Case Report & $4 / 21$ & Rome, Italy & 1 & 78 & Female & & & & & & & & \\
\hline Sohal [95] & Case Report & $5 / 1$ & NY, United States & 1 & 72 & Male & & & & & & & & \\
\hline Zanin [96] & Case Report & $5 / 4$ & Brescia, Italy & 1 & 54 & Female & & & & & & & & \\
\hline $\begin{array}{c}\text { Paniz-Mondolfi } \\
{[97]}\end{array}$ & Case Report & $4 / 21$ & $\begin{array}{l}\text { New York, united } \\
\text { states }\end{array}$ & 1 & 74 & Male & & & & & & & & \\
\hline
\end{tabular}




\begin{tabular}{|c|c|c|c|c|c|c|c|c|c|c|c|c|c|c|c|}
\hline $\begin{array}{c}\text { González-Pinto } \\
\text { [98] }\end{array}$ & Case Report & $4 / 30$ & Bilbao, Spain & 1 & 36 & Female & & & & & & & & & \\
\hline Zhou [99] & Case Report & $4 / 22$ & Xi'an, China & 1 & 75 & Female & & & & & & & & & \\
\hline $\begin{array}{c}\text { Valderrama } \\
{[100]}\end{array}$ & Case Report & $5 / 12$ & $\begin{array}{l}\text { New York, united } \\
\text { states }\end{array}$ & 1 & 52 & Male & & & & & & & & & \\
\hline Viguier [101] & Case Report & $5 / 4$ & Toulouse, France & 1 & 66 & Male & & & & & & & & & \\
\hline Hughes [102] & Case Report & $4 / 29$ & Wales, UK & 1 & 59 & Male & & & & & & & & & \\
\hline $\begin{array}{c}\text { Sharifi-Razavi } \\
{[103]}\end{array}$ & Case Report & $3 / 27$ & Sari, Iran & 1 & 79 & Male & & & & & & & & & \\
\hline Toscao [104] & case report & $4 / 17$ & Pavia, Italy & 4 & - & 4 male & & & & & 4 & 1 & 1 & & 2 \\
\hline Lapergue [105] & case report & $4 / 1$ & Paris, France & 6 & 51.8 & 2-Apr & & & & & & & & & \\
\hline Zhao [106] & case report & $3 / 18$ & Wuhan, China & 1 & 66 & Male & & & & & 1 & & & & \\
\hline Zhang [107] & case report & $4 / 21$ & Wuhan, China & 1 & 40 & Female & & 1 & & & 1 & & & & \\
\hline $\begin{array}{c}\text { Gutierrez Ortiz } \\
{[108]}\end{array}$ & case report & $4 / 17$ & Madrid, Spain & 2 & 50,39 & 2 Male & & 1 & & & & & & & 2 \\
\hline Padroni [109] & case report & $4 / 24$ & Italy & 1 & 70 & Female & & & & & & & & & \\
\hline Poyiadi [110] & case report & $3 / 31$ & $\begin{array}{l}\text { Detroit, Michigan, } \\
\text { United States }\end{array}$ & 1 & 70 & Female & & & 1 & & & & & & \\
\hline Gandolfini [111] & case report & $4 / 14$ & Parma, Italy & 2 & 75,52 & $1 / 1$ & & & & & 2 & & & & \\
\hline \multicolumn{2}{|c|}{ Total } & & & & 8,753 & & $4329 / 3991$ & $\begin{array}{c}142 \\
(1.60 \%)\end{array}$ & $\begin{array}{c}1137 \\
(13.00 \%)\end{array}$ & $\begin{array}{c}21 \\
(0.20 \%)\end{array}$ & $\begin{array}{c}66 \\
(0.75 \%)\end{array}$ & $\begin{array}{c}3300 \\
(37.70 \%)\end{array}$ & $\begin{array}{c}379 \\
(4.30 \%)\end{array}$ & $\begin{array}{c}545 \\
(6.20 \%)\end{array}$ & $\begin{array}{c}646 \\
(7.40 \%)\end{array}$ \\
\hline
\end{tabular}

symptoms were muscle pain (e.g. ache, fatigue, and myalgia) (3300 cases, 53\%), hypogeusia (646 cases, $11 \%$ ), anosmia (545 cases, $9 \%$ ), and hyposmia ( 379 cases, $6 \%$ ) out of the 8,753 reviewed COVID-19 cases. A much smaller proportion of patients had ageusia (74 cases, $1 \%$ ), cerebrovascular diseases (66 cases, 0.75\%), and impaired consciousness ( 21 cases, $0.2 \%$ ) out of 8,753 reviewed COVID-19 cases (Figure 2).

\section{Discussion}

In our systematic review, we showed that the majority of COVID-19 patients have experience in CNS manifestations, including headache and dizziness. Also, common PNS symptoms indicated from our systematic review include muscle pain, hypogeusia, anosmia, and hyposmia. The neurological complication followed by the SARSCOV-2 infection is a critical concern because this viral infection can cause long-lasting brain injury. To date, several studies reported neurological manifestations in COVID-19 patients [1,10,11]. This study confirms that neurological manifestations are associated with SARS-COV-2 infection. It is still unclear how SARS-COV-2 causes neurological complications [12]. It is thought that the SARS-COV-2 virus may enter into the CNS through different mechanisms, such as intranasal inoculation, infected monocytes, interaction with ACE2 receptors on brain endothelial cells, and trans-synaptic transmission [13]. Explanation of the neuroinvasive potential of SARS-COV-2 needs more evidence. However, conducting a systematic analysis of observational studies can get us a precious clue to find out the correlation between neurological symptoms and SARS-COV-2. To this point, we comprehensively reviewed 93 studies with 8,753 cases. In comparison with previous studies, more articles and patients were included and analyzed in the current study $[14,15]$. In agreement with previous studies, neurological symptoms revealed from our systematic review include headache, dizziness, hyposmia, and hypogeusia $[14,16]$. Little is known regarding the pathophysiology of headache in COVID-19 patients. A possible explanation for this might be that cytokine and chemokine production significantly increased by macrophages during the disease [12]. Furthermore, the increase of serum pro-inflammatory cytokines has been suggested for inducing

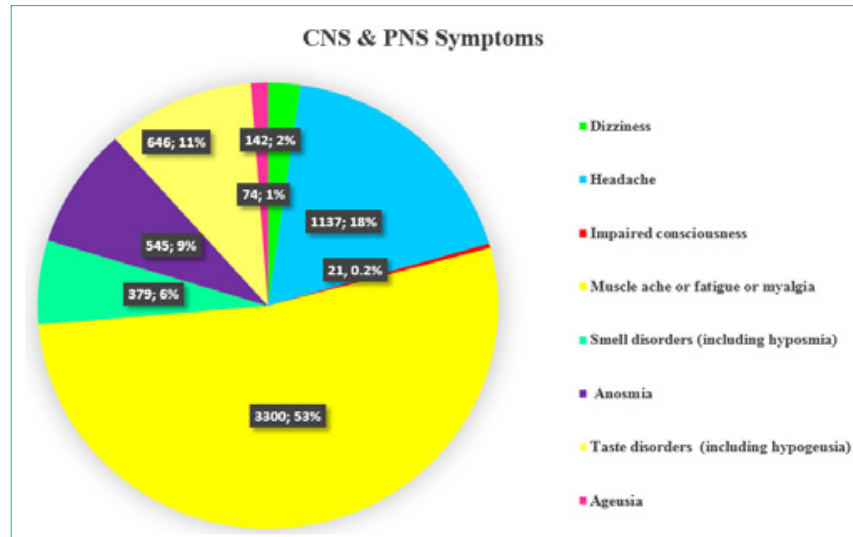

Figure 2: Central Nervous System (CNS) and Peripheral Nervous System (PNS) symptoms of patients infected with COVID-19.

muscle ache, fatigue, and myalgia in COVID-19 patients, which are the most frequently reported PNS manifestations according to our review [1]. Due to the inflammatory mechanism of this virus, there are some other possible disabilities and diseases which can occur during the infection [17-19]. Epilepsy and seizures may occur through Tumor Necrosis Factor (TNF) release and glutamate receptor activation [20]. Some other problems like Guillain-Barre syndrome, meningitis, and encephalitis have also been reported in some cases $[21,11]$.

\section{Conclusion}

To summarize, broad-spectrum of neurological manifestations in both CNS and PNS have been reported. Among these, headache and dizziness are of the top in CNS symptoms and muscle ache, fatigue, and myalgia are the most prevalent in PNS problems. However, some possible pathophysiology of these complications has been suggested, the exact mechanism of the SARS-COV-2 neuroinvasion is not fully understood. Further observational research in this field with more participants would be helpful in the detection of neurological manifestations followed by SARS-COV-2 infection.

Source of Funding 
This study was supported by the Student Research Committee, Mashhad University of Medical Sciences, Mashhad, Iran.

\section{Availability of Data and Materials}

All data and materials are available from the corresponding authors upon request.

\section{Author Contributions}

N.J, N.K, and M.S.G performed search strategy, data extraction, and drew the preliminary tables. S.Y and A.S contributed to the interpretation of the results. E.S and V.H contributed to the analysis of the results. S.S.N conceived of the presented idea and took the lead in writing the manuscript. All authors provided critical feedback and helped shape the research, analysis and manuscript.

\section{References}

1. Mao L, Jin H, Wang M, Hu Y, Chen S, He Q, et al. Neurologic Manifestations of Hospitalized Patients With Coronavirus Disease 2019 in Wuhan, China. JAMA Neurol. 2020

2. Li MY, Li L, Zhang Y, Wang XS. Expression of the SARS-CoV-2 cell receptor gene ACE2 in a wide variety of human tissues. Infect Dis Poverty. 2020; 9 45.

3. Chen X, Laurent S, Onur OA, Kleineberg NN, Fink GR, Schweitzer F, et al. A systematic review of neurological symptoms and complications of COVID-19. J Neurol. 2020; 1-11.

4. Chen R, Yu J, Wang K, Howard D, French L, Chen Z, et al. The spatial and cell-type distribution of SARS-CoV-2 receptor ACE2 in human and mouse brain. BioRxiv. 2020.

5. Chen L, Li X, Chen M, Feng Y, Xiong C. The ACE2 expression in human heart indicates new potential mechanism of heart injury among patients infected with SARS-CoV-2. Cardiovasc Res. 2020; 116: 1097-1100.

6. Liu K, Pan M, Xiao Z, Xu X. Neurological manifestations of the coronavirus (SARS-CoV-2) pandemic 2019-2020. J Neurol Neurosurg Psychiatry. 2020

7. Wu Y, Xu X, Chen Z, Duan J, Hashimoto K, Yang L, et al. Nervous system involvement after infection with COVID-19 and other coronaviruses. Brain Behav Immun. 2020.

8. Yan $\mathrm{CH}$, Faraji F, Prajapati DP, Boone CE, DeConde AS. Association of chemosensory dysfunction and Covid-19 in patients presenting with influenza-like symptoms. Int Forum Allergy Rhinol. 2020.

9. World Health O. Clinical management of COVID-19: interim guidance, 27 May 2020. World Health Organization, Geneva. 2020.

10. Ferrarese C, Silani V, Priori A, Galimberti S, Agostoni E, Monaco S, et al. An Italian multicenter retrospective-prospective observational study on neurological manifestations of COVID-19 (NEUROCOVID). Neurological sciences: official journal of the Italian Neurological Society and of the Italian Society of Clinical Neurophysiology. 2020; 41: 1355-1359.

11. Moriguchi T, Harii N, Goto J, Harada D, Sugawara H, Takamino J, et al. A first case of meningitis/encephalitis associated with SARS-Coronavirus-2. Int J Infect Dis. 2020; 94: 55-58.

12. Zubair AS, McAlpine LS, Gardin T, Farhadian S, Kuruvilla DE, Spudich S Neuropathogenesis and Neurologic Manifestations of the Coronaviruses in the Age of Coronavirus Disease 2019: A Review. JAMA Neurol. 2020; 77 1018-1027.

13. Sepehrinezhad A, Shahbazi A, Negah SS. COVID-19 virus may have neuroinvasive potential and cause neurological complications: a perspective review. Journal of neurovirology. 2020; 26: 324-329.

14. Nepal G, Rehrig JH, Shrestha GS, Shing YK, Yadav JK, Ojha R, et al Neurological manifestations of COVID-19: a systematic review. Crit Care. 2020; 24: 421.

15. Correia AO, Feitosa PWG, Moreira JLS, Nogueira SÁR, Fonseca RB, Nobre
MEP. Neurological manifestations of COVID-19 and other coronaviruses: A systematic review. Neurology, psychiatry, and brain research. 2020; 37: 27-32.

16. Ahmad I, Rathore FA. Neurological manifestations and complications of COVID-19: A literature review. J Clin Neurosci. 2020; 77: 8-12.

17. Jiang L, Tang K, Levin M, Irfan O, Morris SK, Wilson K, et al. COVID-19 and multisystem inflammatory syndrome in children and adolescents. Lancet Infect Dis. 2020

18. Del Valle DM, Kim-Schulze S, Huang H-H, Beckmann ND, Nirenberg S, Wang B, et al. An inflammatory cytokine signature predicts COVID-19 severity and survival. Nature Medicine. 2020.

19. Tian D, Ye Q. Hepatic complications of COVID-19 and its treatment. J Med Virol. 2020; 92: 1818-1824.

20. Niazkar HR, Zibaee B, Nasimi A, Bahri N. The neurological manifestations of COVID-19: a review article. Neurol Sci. 2020; 41: 1667-1671.

21. Camdessanche JP, Morel J, Pozzetto B, Paul S, Tholance Y, BotelhoNevers E. COVID-19 may induce Guillain-Barre syndrome. Rev Neurol (Paris). 2020.

22. Gupta N, Agrawal S, Ish P, Mishra S, Gaind R, Usha G, et al. Clinical and epidemiologic profile of the initial COVID-19 patients at a tertiary care centre in India. Monaldi Archives for Chest Disease. 2020; 90.

23. Ashraf MA, Shokouhi N, Shirali E, Davari-tanha F, Memar O, Kamalipour A, et al. COVID-19 in Iran, a comprehensive investigation from exposure to treatment outcomes. 2020

24. Huang C, Wang Y, Li X, Ren L, Zhao J, Hu Y, et al. Clinical features of patients infected with 2019 novel coronavirus in Wuhan, China. The lancet. 2020; 395: 497-506.

25. Jin X, Lian J-S, Hu J-H, Gao J, Zheng L, Zhang Y-M, et al. Epidemiological, clinical and virological characteristics of 74 cases of coronavirus-infected disease 2019 (COVID-19) with gastrointestinal symptoms. Gut. 2020; 69: 1002-1009

26. Kong I, Park Y, Woo Y, Lee J, Cha J, Choi J, et al. Early epidemiological and clinical characteristics of 28 cases of coronavirus disease in South Korea. Osong Public Health Res Perspect. 2020; 11: 8-14.

27. Liu K, Fang Y-Y, Deng Y, Liu W, Wang M-F, Ma J-P, et al. Clinical characteristics of novel coronavirus cases in tertiary hospitals in Hubei Province. Chinese medical journal. 2020.

28. Chen X, Liu Z. Early prediction of mortality risk among severe COVID-19 patients using machine learning. 2020.

29. Lu X, Gong W, Wang L, Li L, Xie B, Peng Z, et al. Clinical features and high resolution CT imaging findings of preliminary COVID-19. Chinese Journal of Radiology (China). 2020; 54.

30. Qin C, Zhou L, Hu Z, Zhang S, Yang S, Tao Y, et al. Dysregulation of immune response in patients with COVID-19 in Wuhan, China. Clinical Infectious Diseases. 2020.

31. Tian S, Hu N, Lou J, Chen K, Kang X, Xiang Z, et al. Characteristics of COVID-19 infection in Beijing. Journal of Infection. 2020.

32. Yan CH, Faraji F, Prajapati DP, Ostrander BT, DeConde AS. Self-reported olfactory loss associates with outpatient clinical course in COVID-19. In: International Forum of Allergy \& Rhinology. 2020.

33. Zhang R, Ouyang $\mathrm{H}$, Fu L, Wang S, Han J, Huang K, et al. CT features of SARS-CoV-2 pneumonia according to clinical presentation: a retrospective analysis of 120 consecutive patients from Wuhan city. European radiology. 2020; $1-10$

34. Beltrán-Corbellini Á, Chico-García JL, Martínez-Poles J, Rodríguez-Jorge F, Natera-Villalba E, Gómez-Corral J, et al. Acute-onset smell and taste disorders in the context of COVID-19: a pilot multicentre polymerase chain reaction based case-control study. European journal of neurology. 2020.

35. Bénézit $F$, Le Turnier $P$, Declerck $C$, Paillé $C$, Revest $M$, Dubée $V$, et al. Utility of hyposmia and hypogeusia for the diagnosis of COVID-19. The 
Lancet Infectious Diseases. 2020.

36. Ge Y, Tian T, Huang S, Wan F, Li J, Li S, et al. A data-driven drug repositioning framework discovered a potential therapeutic agent targeting COVID-19. 2020.

37. Chen T, Wu D, Chen H, Yan W, Yang D, Chen G, et al. Clinical characteristics of 113 deceased patients with coronavirus disease 2019: retrospective study. 2020; 368.

38. Klopfenstein T, Toko L, Royer P-Y, Lepiller Q, Gendrin V, Zayet S. Features of anosmia in COVID-19. Médecine et Maladies infectieuses. 2020.

39. Lechien JR, Cabaraux P, Chiesa-Estomba C, Khalife M, Plzak J, Hans S Objective olfactory testing in patients presenting with sudden onset olfactory dysfunction as the first manifestation of confirmed COVID-19 infection. 2020

40. Lechien JR, Chiesa-Estomba CM, De Siati DR, Horoi M, Le Bon SD Rodriguez $\mathrm{A}$, et al. Olfactory and gustatory dysfunctions as a clinical presentation of mild-to-moderate forms of the coronavirus disease (COVID-19): a multicenter European study. European Archives of OtoRhino-Laryngology. 2020; 1-11

41. Wang Z, Yang B, Li Q, Wen L, Zhang R. Clinical Features of 69 Cases With Coronavirus Disease 2019 in Wuhan, China. Clin Infect Dis. 2020.

42. Vaira LA, Deiana G, Fois AG, Pirina P, Madeddu G, De Vito A, et al. Objective evaluation of anosmia and ageusia in COVID-19 patients: Singlecenter experience on 72 cases. Head \& neck. 2020; 42: 1252-1258.

43. Jia $\mathrm{X}$, Zhang $\mathrm{P}$, Tian $\mathrm{Y}$, Wang J, Zeng $\mathrm{H}$, Wang J, et al. Clinical significance of IgM and IgG test for diagnosis of highly suspected COVID-19 infection. 2020.

44. Khalili M, Karamouzian M, Nasiri N, Javadi S, Mirzazadeh A, Sharifi H Epidemiological Characteristics of COVID-19: A Systemic Review and MetaAnalysis. 2020.

45. Moein ST, Hashemian SM, Mansourafshar B, Khorram-Tousi A, Tabarsi P, Doty RL. Smell dysfunction: a biomarker for COVID-19. In: International forum of allergy \& rhinology. Wiley Online Library. 2020.

46. Tostmann A, Bradley J, Bousema T, Yiek W-K, Holwerda M, BleekerRovers $C$, et al. Strong associations and moderate predictive value of early symptoms for SARS-CoV-2 test positivity among healthcare workers, the Netherlands, March 2020. Eurosurveillance. 2020; 25: 2000508

47. lechien J, cabaraux $P$, chiesa-estomba C, khalife M, plzak J, hans S, et al. Objective olfactory testing in patients presenting with sudden onset olfactory dysfunction as the first manifestation of confirmed COVID-19 infection. 2020

48. Liu M, He P, Liu H, Wang X, Li F, Chen S, et al. Clinical characteristics of 30 medical workers infected with new coronavirus pneumonia. Zhonghua jie he he hu xi za zhi= Zhonghua jiehe he huxi zazhi= Chinese journal of tuberculosis and respiratory diseases. 2020; 43: E016-E016.

49. Liao $\mathrm{Y}$, Feng $\mathrm{Y}$, Wang $\mathrm{B}$, Wang $\mathrm{H}$, Huang $\mathrm{J}$, Wu $\mathrm{Y}$, et al. Clinical Characteristics and Risk factors for developed COVID-19 patients transferring to designated hospital from Jianghan Fangcang shelter Hospital: a retrospective, observational study. 2020.

50. Chen N, Zhou M, Dong X, Qu J, Gong F, Han Y, et al. Epidemiological and clinical characteristics of 99 cases of 2019 novel coronavirus pneumonia in Wuhan, China: a descriptive study. The Lancet. 2020; 395: 507-513.

51. Ding Q, Lu P, Fan Y, Xia Y, Liu M. The clinical characteristics of pneumonia patients coinfected with 2019 novel coronavirus and influenza virus in Wuhan, China. Journal of medical virology. 2020.

52. Du R-H, Liang L-R, Yang C-Q, Wang W, Cao T-Z, Li M, et al. Predictors of mortality for patients with COVID-19 pneumonia caused by SARS-CoV-2: a prospective cohort study. European Respiratory Journal. 2020; 55.

53. Escalera-Antezana JP, Lizon-Ferrufino NF, Maldonado-Alanoca A, AlarcónDe-la-Vega G, Alvarado-Arnez LE, Balderrama-Saavedra MA, et al. Clinical features of cases and a cluster of Coronavirus Disease 2019 (COVID-19) in Bolivia imported from Italy and Spain. Travel Medicine and Infectious Disease. 2020; 101653.

54. Gilani S, Roditi R, Naraghi M. COVID-19 and anosmia in Tehran, Iran
Medical Hypotheses. 2020; 109757.

55. Wang D, Ju X, Xie F, Lu Y, Li F, Huang H, et al. Clinical analysis of 31 cases of 2019 novel coronavirus infection in children from six provinces (autonomous region) of northern China. Zhonghua er ke za zhi= Chinese journal of pediatrics. 2020; 58: E011-E011.

56. Wang X, Liu W, Zhao J, Lu Y, Wang X, Yu C, et al. Clinical characteristics of 80 hospitalized frontline medical workers infected with COVID-19 in Wuhan, China. Journal of Hospital Infection. 2020.

57. Xu X-W, Wu X-X, Jiang X-G, Xu K-J, Ying L-J, Ma C-L, et al. Clinical findings in a group of patients infected with the 2019 novel coronavirus (SARSCov-2) outside of Wuhan, China: retrospective case series. 2020; 368

58. Yan Y, Yang Y, Wang F, Ren H, Zhang S, Shi X, et al. Clinical characteristics and outcomes of patients with severe covid-19 with diabetes. BMJ Open Diabetes Research and Care. 2020; 8: e001343.

59. Zhang H, Chen Y, Yuan Q, Xia Q-X, Zeng X-P, Peng J-T, et al. Identification of kidney transplant recipients with coronavirus disease 2019. European Urology. 2020

60. Zhang X, Cai H, Hu J, Lian J, Gu J, Zhang S, et al. Epidemiological, clinical characteristics of cases of SARS-CoV-2 infection with abnormal imaging findings. International Journal of Infectious Diseases. 2020.

61. Tang C, Zhang K, Wang W, Pei Z, Liu Z, Yuan P, et al. Clinical Characteristics of 20,662 Patients with COVID-19 in mainland China: A Systemic Review and Meta-analysis. 2020

62. Zhang L, Zhu F, Xie L, Wang C, Wang J, Chen R, et al. Clinical characteristics of COVID-19-infected cancer patients: a retrospective case study in three hospitals within Wuhan, China. Annals of Oncology. 2020.

63. Monti S, Balduzzi S, Delvino P, Bellis E, Quadrelli VS, Montecucco C Clinical course of COVID-19 in a series of patients with chronic arthritis treated with immunosuppressive targeted therapies. Annals of the rheumatic diseases. 2020; 79: 667-668

64. Guan W-J, Ni Z-Y, Hu Y, Liang W-H, Ou C-Q, He J-X, et al. Clinical characteristics of coronavirus disease 2019 in China. New England journal of medicine. 2020; 382: 1708-1720.

65. Zeng L, Li J, Liao M, Hua R, Huang P, Zhang M, et al. Risk assessment of progression to severe conditions for patients with COVID-19 pneumonia: a single-center retrospective study. 2020.

66. Wu C, Chen X, Cai Y, Zhou X, Xu S, Huang H, et al. Risk factors associated with acute respiratory distress syndrome and death in patients with coronavirus disease 2019 pneumonia in Wuhan, China. JAMA internal medicine. 2020.

67. Mo $\mathrm{P}$, Xing $\mathrm{Y}$, Xiao $\mathrm{Y}$, Deng $\mathrm{L}$, Zhao $\mathrm{Q}$, Wang $\mathrm{H}$, et al. Clinical characteristics of refractory COVID-19 pneumonia in Wuhan, China. Clinical Infectious Diseases. 2020.

68. Hintschich CA, Wenzel JJ, Hummel T, Hankir MK, Kühnel T, Vielsmeier V, et al. Psychophysical tests reveal impaired olfaction but preserved gustation in COVID-19 patients. Int Forum Allergy Rhinol. 2020; 10: 1105-1107.

69. Coppola A, Tonini MC, Baratelli E, Barillà C, Bassani R, Gonano EF, et al. Headache in a group of SARS-COVID-19 patients: an observational prospectical study. Neurol Sci. 2020; 1-2.

70. Klok F, Kruip M, Van der Meer N, Arbous M, Gommers D, Kant K, et al. Incidence of thrombotic complications in critically ill ICU patients with COVID-19. Thrombosis research. 2020

71. Oxley TJ, Mocco J, Majidi S, Kellner CP, Shoirah H, Singh IP, et al. LargeVessel Stroke as a Presenting Feature of Covid-19 in the Young. New England Journal of Medicine. 2020; 382: e60.

72. Helms J, Kremer S, Merdji H, Clere-Jehl R, Schenck M, Kummerlen C, et al Neurologic features in severe SARS-CoV-2 infection. New England Journal of Medicine. 2020

73. Garg R, Jain R, Sodani A, Chouksey D, Dosi R, Athale S, et al. Neurological symptoms as initial manifestation of Covid-19-An observational study. Ann Indian Acad Neurol. 2020; 23: 482-486. 
74. Jang Y, Son H-J, Lee S, Lee EJ, Kim TH, Park SY. Olfactory and taste disorder: the first and only sign in a patient with SARS-CoV-2 pneumonia. Infection Control \& Hospital Epidemiology. 2020; 1-1.

75. Ollarves-Carrero MF, Rodriguez-Morales AG, Bonilla-Aldana DK Rodriguez-Morales AJ. Anosmia in a healthcare worker with COVID-19 in Madrid, Spain. Travel Med Infect Dis. 2020; 101666.

76. Zhao H, Shen D, Zhou H, Liu J, Chen S. Guillain-Barre syndrome associated with SARS-CoV-2 infection: causality or coincidence? Lancet Neurol. 2020; 19: 383-384.

77. Sedaghat Z, Karimi N. Guillain Barre syndrome associated with COVID-19 infection: A case report. J Clin Neurosci. 2020.

78. El Otmani H, El Moutawakil B, Rafai M-A, El Benna N, El Kettani C, Souss M, et al. Covid-19 and Guillain-Barré syndrome: More than a coincidence! Rev Neurol. 2020.

79. Huang YH, Jiang D, Huang JT (2020) A Case of COVID-19 Encephalitis. Brain Behav Immun

80. Duong L, Xu P, Liu A (2020) Meningoencephalitis without respiratory failure in a young female patient with COVID-19 infection in Downtown Los Angeles, early April 2020. Brain Behav Immun

81. Ye M, Ren Y, Lv T. Encephalitis as a clinical manifestation of COVID-19. Brain Behav Immun. 2020.

82. Yin R, Feng W, Wang T, Chen G, Wu T, Chen D, et al. Concomitan neurological symptoms observed in a patient diagnosed with coronavirus disease 2019. J Med Virol. 2020.

83. Filatov A, Sharma P, Hindi F, Espinosa PS. Neurological complications of coronavirus disease (COVID-19): encephalopathy. Cureus. 2020; 12.

84. Wong PF, Craik S, Newman P, Makan A, Srinivasan K, Crawford E, et al Lessons of the month 1: A case of rhombencephalitis as a rare complication of acute COVID-19 infection. Clinical Medicine. 2020; 20: 293-294.

85. Virani A, Rabold E, Hanson T, Haag A, Elrufay R, Cheema T, et al. GuillainBarré syndrome associated with SARS-CoV-2 infection. 2020.

86. Alberti $P$, Beretta $S$, Piatti $M$, Karantzoulis A, Piatti ML, Santoro $P$, et al. Guillain-Barré syndrome related to COVID-19 infection. NeurologyNeuroimmunology Neuroinflammation. 2020; 7.

87. Scheidl E, Canseco DD, Hadji-Naumov A, Bereznai B. Guillain-Barré syndrome during SARS-CoV-2 pandemic: A case report and review of recent literature. Journal of the Peripheral Nervous System. 2020; 25: 204-207.

88. Ottaviani D, Boso F, Tranquillini E, Gapeni I, Pedrotti G, Cozzio S, et al. Early Guillain-Barré syndrome in coronavirus disease 2019 (COVID-19): a case report from an Italian COVID-hospital. Neurol Sci. 2020; 1.

89. Abdelnour L, Eltahir Abdalla M, Babiker S. COVID 19 infection presenting as motor peripheral neuropathy. J Formos Med Assoc. 2020; 119: 1119-1120.

90. Juliao Caamaño DS, Alonso Beato R. Facial diplegia, a possible atypical variant of Guillain-Barré Syndrome as a rare neurological complication of SARS-CoV-2. J Clin Neurosci. 2020; 77: 230-232.

91. Wei $\mathrm{H}$, Yin $\mathrm{H}$, Huang M, Guo Z. The 2019 novel cornoavirus pneumonia with onset of oculomotor nerve palsy: a case study. J Neurol. 2020; 1-4.

92. Jin M, Tong Q. Rhabdomyolysis as Potential Late Complication Associated with COVID-19. Emerg Infect Dis. 2020; 26: 1618-1620.

93. Suwanwongse K, Shabarek N. Rhabdomyolysis as a presentation of 2019 novel coronavirus disease. Cureus. 2020; 12.
94. Vollono C, Rollo E, Romozzi M, Frisullo G, Servidei S, Borghetti A, et al. Focal status epilepticus as unique clinical feature of COVID-19: A case report. Seizure. 2020; 78: 109-112.

95. Sohal S, Mansur M. COVID-19 Presenting with Seizures. 2020; 20: e00782.

96. Zanin L, Saraceno G, Panciani PP, Renisi G, Signorini L, Migliorati K, et al. SARS-CoV-2 can induce brain and spine demyelinating lesions. Acta Neurochirurgica. 2020; 1-4

97. Paniz-Mondolfi A, Bryce C, Grimes Z, Gordon RE, Reidy J, Lednicky J, et al. Central Nervous System Involvement by Severe Acute Respiratory Syndrome Coronavirus -2 (SARS-CoV-2). J Med Virol. 2020.

98. González-Pinto T, Luna-Rodríguez A, Moreno-Estébanez A, Agirre-Beitia G, Rodríguez-Antigüedad A, Ruiz-Lopez M. Emergency Room Neurology in times of COVID-19: Malignant Ischemic Stroke and SARS-CoV-2 Infection. Eur J Neurol. 2020.

99. Zhou B, She J, Wang Y, Ma X. A Case of Coronavirus Disease 2019 With Concomitant Acute Cerebral Infarction and Deep Vein Thrombosis. Frontiers in neurology. 2020; 11: 296-296.

100. Valderrama EV, Humbert K, Lord A, Frontera J, Yaghi S. Severe Acute Respiratory Syndrome Coronavirus 2 Infection and Ischemic Stroke. Stroke. 2020; 51: e124-e127.

101. Viguier A, Delamarre L, Duplantier J, Olivot JM, Bonneville F. Acute ischemic stroke complicating common carotid artery thrombosis during a severe COVID-19 infection. Journal of neuroradiology. 2020.

102. Hughes C, Nichols T, Pike M, Subbe C, Elghenzai S. Cerebral Venous Sinus Thrombosis as a Presentation of COVID-19. European Journal of Case Reports in Internal Medicine. 2020; 7.

103. Sharifi-Razavi A, Karimi N, Rouhani N. COVID-19 and intracerebral haemorrhage: causative or coincidental? New Microbes New Infect. 2020; 35.

104. Toscano G, Palmerini F, Ravaglia S, Ruiz L, Invernizzi P, Cuzzoni MG, et al. Guillain-Barré syndrome associated with SARS-CoV-2. New England Journal of Medicine. 2020.

105. Lapergue B, Lyoubi A, Meseguer E, Avram I, Denier C, Venditti L, et al. Large vessel stroke in six patients following SARS-CoV-2 infection: a retrospective case study series of acute thrombotic complications on stable underlying atherosclerotic disease. Eur J Neurol. 2020.

106. Zhao K, Huang J, Dai D, Feng Y, Liu L, Nie S. Acute myelitis after SARSCoV-2 infection: a case report. 2020.

107.Zhang T, Rodricks MB, Hirsh E. COVID-19-Associated Acute Disseminated Encephalomyelitis: A Case Report. 2020.

108. Gutierrez-Ortiz C, Mendez A, Rodrigo-Rey S, San Pedro-Murillo E, BermejoGuerrero L, Gordo-Manas R, et al. Miller Fisher Syndrome and polyneuritis cranialis in COVID-19. Neurology. 2020.

109. Padroni M, Mastrangelo V, Asioli GM, Pavolucci L, Abu-Rumeileh S, Piscaglia MG, et al. Guillain-Barre syndrome following COVID-19: new infection, old complication? J Neurol. 2020.

110. Poyiadji N, Shahin G, Noujaim D, Stone M, Patel S, Griffith B. COVID-19associated acute hemorrhagic necrotizing encephalopathy: CT and MRI features. Radiology. 2020.

111. Gandolfini I, Delsante M, Fiaccadori E, Zaza G, Manenti L, Degli Antoni A, et al. COVID-19 in kidney transplant recipients. American Journal of Transplantation. 2020. 